\title{
Development of Depressive Symptoms During Early Community Reintegration After Traumatic Brain Injury
}

\author{
Tamara Ownsworth, ${ }^{1}$ Jennifer Fleming, ${ }^{2,3}$ Terry Haines, ${ }^{4,5}$ Petrea Cornwell, ${ }^{1,6}$ Melissa Kendall, ${ }^{7}$ \\ Emily Nalder, ${ }^{2,3}$ AND Cassandra Gordon ${ }^{1}$ \\ ${ }^{1}$ School of Psychology and Griffith Institute for Health and Medical Research, Griffith University, Mt Gravatt, Australia \\ ${ }^{2}$ The University of Queensland, School of Health and Rehabilitation Sciences, St Lucia, Australia \\ ${ }^{3}$ Occupational Therapy Department, Princess Alexandra Hospital, Queensland, Australia \\ ${ }^{4}$ School of Physiotherapy, Monash University, Victoria, Australia \\ ${ }^{5}$ Allied Health Clinical Research Unit, Southern Health, Victoria, Australia \\ ${ }^{6}$ Metro North Health Service District, Queensland Health, Australia \\ ${ }^{7}$ Acquired Brain Injury Outreach Service, Princess Alexandra Hospital, Buranda, Australia
}

(Received June 16, 2010; Final Revision September 22, 2010; AcCePted September 23, 2010)

\begin{abstract}
The early onset of depression following traumatic brain injury (TBI) is associated with poorer psychosocial outcomes; however, the direction of this relationship is unclear. This study investigated the relationship between progress in resuming preinjury lifestyle (transition events), change in perceived functioning and level of depressive symptoms at discharge and 3-months postdischarge. As part of a prospective longitudinal study of brain injury outcomes, 96 consecutively discharged patients with TBI completed measures of transition events (Sentinel Events Questionnaire) and perceived functioning (Mayo-Portland Adaptability Inventory-4 Ability and Adjustment indices) at discharge and 3-months follow-up. Level of depressive symptoms was assessed at discharge and 3-months follow-up using the Depression Anxiety Stress Scale 21. After controlling for age and discharge depressive symptoms, change in perceived functioning was found to mediate the relationship between total transition events and depressive symptoms at 3-months postdischarge ( $\beta$ reduced from .21 to .14), with a significant indirect effect observed. The present findings indicate that lack of progress in resuming preinjury lifestyle contributes to postdischarge depressive symptoms through an influence on perceived functioning, thus providing an improved conceptualization of reactive depression in the context of brain injury. (JINS, 2011, 17, 112-119)
\end{abstract}

Keywords: Traumatic brain injury, Depressive symptoms, Community reintegration, Perceived functioning, Transition events

\section{INTRODUCTION}

Traumatic brain injury (TBI) is a major global health concern affecting approximately 10 million new people each year worldwide. The majority are young adults in the process of establishing their independent social and working lives (Corrigan, Selassie, \& Orman, 2010). Moderate to severe TBI typically results in complex physical, cognitive and behavioral impairments that can greatly reduce individuals' capacity for independent living and employment and the ability to establish and maintain relationships (Tate \& Broe, 1999). Depression is common following TBI with prevalence rates most typically between 26 and $42 \%$ (Kreutzer, Seel, \&

Correspondence and reprint requests to: Tamara Ownsworth, School of Psychology, Griffith University, Mt Gravatt, 4122 Australia. E-mail: t.ownsworth@griffith.edu.au
Gourley, 2001). Multiple etiological pathways have been proposed for post-TBI depression, including the exacerbation of a preexisting psychological disorder or vulnerability (Ownsworth \& Oei, 1998), disruption of corticolimbic circuitry that mediates emotional regulation (Jorge et al., 2004), and a psychological reaction to changes in personal abilities and social opportunities (Godfrey, Partridge, Knight, \& Bishara, 1993). As no single explanation can adequately account for the varied clinical presentations, there is a general consensus in the literature that depression following TBI arises from a complex interplay between neurological, psychological, and social factors (Williams \& Evans, 2003).

The early onset of depression after TBI has been linked to poorer community reintegration outcomes, including unemployment (Felmingham, Baguley, \& Crooks, 2001; Ruff et al., 1993) and impaired close relationships (Gomez-Hernandez, Max, Kosier, Paradiso, \& Robinson, 1997). Depression is 
also found to be related to reduced independence and poorer interpersonal functioning and productivity in the long-term (Ownsworth \& Fleming, 2005). However, the direction of the relationship between depression and psychosocial outcome is largely unclear. Specifically, depression may precede and contribute to poor psychosocial outcomes, or be a consequence of postinjury limitations, such as the inability to resume preinjury activities (e.g., driving, work, independent living) and financial strain (Seel et al., 2003). A clearer understanding of this relationship is needed to guide the focus of early psychological interventions, for which an evidence base is currently lacking (Bombardier et al., 2009). Accordingly, the present study focuses on events during the hospital-to-home transition and perceived functional consequences as predictors of depressive symptoms during early community reintegration.

As individuals leave the hospital environment and are faced with the impact of their TBI-related impairments at home, they often begin to better comprehend how the injury will affect their independence, relationships, work, and future plans (Godfrey et al., 1993; Turner, Ownsworth, Cornwell, \& Fleming, 2009). Changes in personal abilities and lifestyle restrictions (e.g., inability to drive or work) can threaten sense of self and social identity. Access to therapy during this transition period is therefore viewed as critical to support individuals' goals for community reintegration (Gracey, Evans \& Malley, 2009). Malec, Testa, Rush, Brown, and Moessner (2007) found that self-assessed impairment was the best predictor of depression at discharge and "late" depression at $1-2$ years postinjury $(n=84)$. Levels of depression did not differ between TBI and orthopedic patients or between those with mild and moderate to severe injury TBI. The authors concluded that subjective impairment had a more direct effect on depressive symptoms than neurological factors. By focusing on perceived impairment only at the time of discharge, however, their study did not capture the changes in functioning that individuals often perceive after hospital. Furthermore, depression at discharge was not controlled for in the analysis of late depressive symptoms.

Lifestyle stressors have been found to influence emotional adjustment in long-term TBI (Rutterford \& Wood, 2006); however, the emotional impact of early transition events after TBI has been largely overlooked. Research by Turner et al. (2009) identified that individuals with brain injury viewed the hospital-to-home transition more favorably when greater progress was made in resuming their preinjury lifestyle. Several key transition events were perceived as impacting progress in resuming preinjury lifestyle during the first 3-months postdischarge. Specifically, return to work, driving, home independence, and community access represent progress in resuming preinjury lifestyle. Conversely, financial strain, relationship breakdown, changed living situation, and lack of access to therapy reflect difficulties or barriers to resuming or maintaining preinjury lifestyle (Turner et al., 2009). Individuals' perception of their postinjury selves is shaped by their attempts to resume or maintain their preinjury lifestyle (Gracey et al., 2009; Ownsworth \& Oei, 1998). Transition events are therefore expected to contribute to level of depressive symptoms following discharge through their impact on perceived functioning.

The present study aimed to determine the relationship between transition events (i.e., progress in resuming preinjury lifestyle), perceived functioning and depressive symptoms during early community reintegration following TBI. It was hypothesized that change in perceived functioning between discharge and 3-months follow-up would significantly mediate the relationship between transition events and depressive symptoms at 3-months postdischarge.

\section{METHODS}

\section{Participants}

Participants with TBI were recruited as part of a larger prospective longitudinal study of acquired brain injury hospital transition outcomes. This broader study involved the consecutive recruitment of 204 patients with acquired brain injury (including TBI, stroke, hypoxia) discharged from the inpatient Brain Injury Rehabilitation Unit and acute neurosciences ward of a major metropolitan hospital between September 2007 and July 2009. Participants were eligible for the study if they had sustained a TBI from any cause, were aged 18-60 years, were hospitalized for at least 4 days before being discharged home, and displayed adequate English communication skills. A total of 196 eligible people with TBI were discharged during this period, and 129 individuals $(65.82 \%)$ were recruited. The 67 eligible individuals who declined participation were significantly younger $(p<.01)$, but did not differ in terms of gender, cause of TBI and length of hospitalization $(p>.05)$. There were missing data regarding level of functioning on key measures at discharge for 11 participants with TBI. Additionally, 22 participants were either unable to be contacted or withdrew from the study at the 3-months postdischarge follow-up. Therefore, 96 of 129 consenting individuals with TBI (74.4\%) completed all discharge and 3-month follow-up assessments. A comparison between those who completed all assessments and those with missing data at follow-up revealed no significant differences concerning age, gender, education, injury severity, disability severity and discharge depressive symptoms $(p>.05)$.

Table 1 presents a summary of the demographic and injury characteristics of the $96 \mathrm{TBI}$ participants. The majority of the sample were male $(81 \%)$, sustained their TBI in a trafficrelated accident $(48 \%)$ and, on average, participants were hospitalized for 57 days $(S D=53.91)$. At the time of injury, $50 \%$ were married or in a relationship and $94 \%$ were in fulltime or part-time employment/study ( $6 \%$ were homemakers). The majority of the sample identified themselves as Caucasian (92\%). Data concerning Glasgow Coma Scale (GCS) and duration of posttraumatic amnesia (PTA) were retrieved from medical records. Based on these indices and standard classification criteria (see Kolb \& Whishaw, 2003), 12\% had sustained a mild-moderate TBI (GCS 9-15 or PTA $<24 \mathrm{hr}$ ) and $88 \%$ had sustained a severe TBI (GCS $<9$ or PTA $>24 \mathrm{hr}$ ). 
Table 1. Descriptive data for the traumatic brain injury sample $(n=96)$

\begin{tabular}{lc}
\hline \hline Variable & $\begin{array}{c}\text { TBI participants } \\
M(S D), \text { range/N(\%) }\end{array}$ \\
\hline Age (years) & $35.37(13.07), 18-60$ \\
Gender & $78(81.3)$ \\
$\quad$ Male & $18(18.7)$ \\
Female & $12.62(2.72), 8-21$ years \\
Education (years) & $57.67(53.9), 4-270$ \\
Length of hospital stay (days) & $9.15(4.29), 3-15$ \\
Glasgow Coma Scale (initial) & $29.44(31.4),<1$ hour -173 days \\
Posttraumatic amnesia & $3.87(1.65), 1-6$ \\
Disability Rating Scale & \\
$\quad$ discharge) & \\
Cause of TBI & $46(47.9)$ \\
Traffic related & $26(27.1)$ \\
Fall & $17(17.7)$ \\
Assault & $7(7.3)$ \\
Sporting injury & \\
\hline \hline
\end{tabular}

Note. $\mathrm{TBI}=$ traumatic brain injury.

Disability Rating Scale (DRS) scores indicated a mild to moderate level of disability at time of discharge (range: 1-6).

\section{Measures}

The Mayo-Portland Adaptability Inventory-4 (MPAI-4; Malec, 2005) is a standardized tool specifically developed to assess functional consequences of TBI. The 29 items are rated on a 5-point scale $(0=$ no problems in functioning, to $4=$ severe problem that interferes with functioning or activities more than $75 \%$ of the time) and comprise three subscales: abilities, adjustment, and participation. The Ability Index consists of 12 items relating to sensory, motor, communication and cognitive abilities (e.g., vision, mobility, verbal communication, and attention/concentration. The Adjustment Index is comprised of 12 items relating to emotional, behavioral, and social function (e.g., depression, irritability, pain and headache, social interaction). The Participation Index consists of eight items that assess individuals' role participation and their need for assistance and supervision in everyday living (e.g., self-care, transportation, and employment). For each index, raw scores are converted to standard scores (see Malec, 2005) with higher total scores indicating poorer perceived functioning. The MPAI-4 has strong psychometric properties with the total scale and separate indices supported by Rasch analysis (person reliability $=.78-.88$, item reliability $=.98-.99$ ) and Cronbach's alpha (.76-.83; Malec, 2005).

In the present study, although the entire MPAI-4 was administered, only the Ability and Adjustment Indices were used in the analysis due to the key focus on perceived functional consequences of TBI relating to physical, cognitive, emotional and behavioral function. A MPAI-4 change score was derived by summing the standard scores at each time point and calculating a difference score (i.e., Discharge
MPAI-4 [Ability + Adjustment] minus 3-month postdischarge MPAI-4 [Ability + Adjustment]). Using this approach, positive scores reflect perceived improvement in functioning and negative scores reflect poorer perceived functioning relative to discharge.

The Depression, Anxiety, and Stress Scales 21 (DASS-21; Lovibond \& Lovibond, 1995) is a well-validated tool consisting of three 7-item scales from the original 42 -item version. Participants rate on a 4-point scale $(0=$ did not apply to $3=$ applied very much or most of the time) the extent to which they have experienced various mood symptoms over the past week. Items are summed and multiplied by two to derive subscale scores (0-42). A score of 10 or higher on the depression scale indicates a level of symptoms in the clinical range (Lovibond \& Lovibond). The DASS 21 was selected to assess depressive symptoms because items reflect affective and behavioral symptoms of depression, rather than somatic and cognitive symptoms which can overlap with TBI sequelae. The item content of the depression subscale relates to: absence of positive feeling, low initiative, nothing to look forward to, feeling downhearted and blue, unable to feel enthusiastic, worthlessness, and a sense of life being meaningless (Lovibond \& Lovibond, 1995). The DASS 21-depression scale has been found to have sound internal consistency $(\alpha=.88)$, test-retest reliability $(r=.78)$ and validity in brain injury research (Ownsworth, Little, Turner, Hawkes, \& Shum, 2008).

The Sentinel Events Questionnaire (SEQ) was developed by the authors (Nalder et al., 2010; Ownsworth et al., 2009) to assess the occurrence of eight transition events since the brain injury (discharge SEQ) and since the last assessment (3-month follow-up SEQ). Standard questions and prompts are used to identify the presence of the following transition events: (a) financial strain, (b) relationship breakdown, (c) changed living situation, (d) lack of access to therapy, (e) return to driving, (f) return to work/study, (g) community access, and (h) home independence. In this study, a score of $0-8$ was used to indicate total transition events at the time of discharge as well as for the period up to 3-months postdischarge, thus representing overall progress in resuming preinjury lifestyle. Specifically, a score of 1 was assigned for each event present for items $1-4$ and a score of 1 was assigned for each event not present in items 5-8. Therefore, an individual reporting all of the events in items 1-4, but none of the events in items 5-8 would receive a total score of 8 , thus reflecting the poorest overall progress. A high level of agreement (75-100\%) has been found between the reports of individuals with TBI and their caregivers for each item (Ownsworth et al., 2009).

\section{Procedure}

Ethical clearance was granted by hospital and university human ethics committees. Participants were initially screened and approached to participate in the study by their occupational therapist. After providing informed consent, all participants were administered the questionnaires in person within the hospital setting at the time of discharge and over the 
telephone at 3-months follow-up. Data regarding injury characteristics and functional status (therapist ratings on the DRS) were retrieved from hospital records.

\section{Data Analysis}

Following data screening and management procedures, $t$ tests and correlation analyses were used to identify demographic, injury and discharge variables associated with the DASS-21 depression score at 3-month follow-up. To test for a mediating effect of change in perceived functioning on the relationship between total transition events (SEQ at 3-months postdischarge) and DASS-21 depression score at 3-months postdischarge, a series of three regression analyses was conducted in accordance with Baron and Kenny (1986). A Sobel test was used to investigate the significance of an indirect effect (MacKinnon, Warsi, \& Dwyer, 1995).

\section{RESULTS}

\section{Comparison Between Measures at Discharge and 3-Months Follow-Up}

As shown in Table 2, level of depressive symptoms on the DASS-21 was in the normal range for the sample at both time points and did not significantly change between discharge and 3-months follow-up $(p>.05)$. The proportion in the clinical range for depression $(\geq 10)$ at discharge and 3-months follow-up was $24 \%$ and $27 \%$, respectively. Classification changed for $23 \%$ of individuals between these assessments, with $11.5 \%$ shifting from the normal range to clinical range, and $11.5 \%$ shifting from the clinical range to normal range. Perceived functioning on the combined Ability and Adjustment
Index of the MPAI-4 did not significantly differ for the overall sample between discharge and 3-months follow-up (change $M=0.84, S D=21.6$ ). However, approximately $47 \%$ perceived their functioning to be at least one point worse on this combined index at follow-up (range: -1 to -61 ) relative to discharge. Greater overall progress in resuming preinjury lifestyle was evident for the sample at 3-months follow-up relative to discharge $(p<.001)$. Specifically, by 3 -months follow-up a significantly higher proportion of individuals had returned to work and driving and had achieved greater independence in the home and community $(p<.001)$.

\section{Mediation Analysis}

A preliminary correlation analysis identified that the DASS-21 depression score at 3-months follow-up was significantly correlated with the discharge DASS-21 depression score $(r=.53, p<.001)$ and age $(r=.28, p<.01)$, but not gender, marital status, education, cause of TBI, severity of injury, length of hospitalization, or DRS at discharge $(p>.05)$. Therefore, age and discharge DASS-21 depression score were treated as covariates in each step of the mediation analysis. Inspection of a correlation matrix of the covariates, total transition events (SEQ at 3-months follow-up), MPAI-4 change score, and DASS-21 depression score at 3-months follow-up (see Table 3 ) confirmed the suitability of mediation analysis (Baron \& Kenny, 1986). Specifically, total transition events significantly correlated with DASS-21 depression score at 3-months $(r=.26)$, total transition events also significantly correlated with MPAI-4 change score $(r=-.21)$, and MPAI-4 change score significantly correlated with DASS-21 depression score at 3 months $(r=-.29)$.

In the first regression analysis (see Table 4), total transition events significantly predicted MPAI-4 change score

Table 2. Comparison of depressive symptoms, perceived functioning, and transition events between discharge and 3-months follow-up

\begin{tabular}{|c|c|c|c|c|c|}
\hline \multirow[b]{2}{*}{ Measure } & \multicolumn{2}{|c|}{$M(S D)$, range/ $N(\%)$} & \multirow[b]{2}{*}{$t / \chi^{2}$} & \multirow[b]{2}{*}{$p$ value } & \multirow[b]{2}{*}{ Effect size $d / P h i$} \\
\hline & Discharge & 3-months & & & \\
\hline DASS 21 depression scale & $5.69(8.15), 0-38$ & 6.49 (8.94), 0-38 & -0.94 & .349 & .09 \\
\hline Normal & $73(76.1 \%)$ & $70(72.9 \%)$ & & & \\
\hline Mild & $8(8.1 \%)$ & $11(11.4 \%)$ & & & \\
\hline Moderate & $10(10.5 \%)$ & $6(6.3 \%)$ & & & \\
\hline Severe & $1(1.1 \%)$ & $3(3.1 \%)$ & & & \\
\hline Very Severe & $4(4.2 \%)$ & $6(6.3 \%)$ & & & \\
\hline MPAI-4 (Ability + Adjustment) & $77.08(21.50)$ & $76.24(22.66)$ & 0.38 & .704 & .04 \\
\hline Change scores & & $0.84(21.56)$ & & & \\
\hline SEQ total scores $(0-8)$ & $3.74(1.0), 2-7$ & $2.2(1.43), 0-6$ & 9.54 & $<.001$ & 1.21 \\
\hline Financial strain & $16(16.67)$ & $19(19.79)$ & & ns & .41 \\
\hline Relationship breakdown & $7(7.29)$ & $7(7.29)$ & & ns & .08 \\
\hline Restrictive living situation & $16(16.67 \%)$ & $17(17.71)$ & & ns & .09 \\
\hline Lack of therapy access & $7(7.29)$ & $15(15.63)$ & & ns & .10 \\
\hline Return to work/study & $4(4.2)$ & $47(48.96)$ & & $<.001$ & .21 \\
\hline Return to driving & $0(0)$ & $29(30.21)$ & & $\mathrm{n} / \mathrm{a}$ & $\mathrm{n} / \mathrm{a}$ \\
\hline Community access & $22(22.9)$ & $65(67.71)$ & & $<.001$ & .22 \\
\hline Home independence & $42(43.75)$ & $86(89.58)$ & & $<.001$ & .10 \\
\hline
\end{tabular}

Note. DASS-21 = Depression Anxiety and Stress Scales 21; MPAI-4 = Mayo-Portland Adaptability Inventory - 4; SEQ = Sentinel Events Questionnaire. 
Table 3. Correlations between age, total transition events, perceived change in functioning, and DASS-21 depression score

\begin{tabular}{|c|c|c|c|c|c|}
\hline Parameter & Age & $\begin{array}{c}\text { Discharge } \\
\text { DASS-21 (D) }\end{array}$ & $\begin{array}{l}\text { MPAI-4 change } \\
\text { (ability/adjustment) }\end{array}$ & $\begin{array}{l}\text { SEQ total } \\
\text { (3-months) }\end{array}$ & $\begin{array}{c}\text { 3-months } \\
\text { DASS-21 (D) }\end{array}$ \\
\hline Age & - & & & & \\
\hline Discharge DASS 21 (D) & .14 & - & & & \\
\hline MPAI-4 change (ability/adjustment) & .05 & .02 & - & & \\
\hline SEQ total (3-months) & .05 & .10 & $-.21 *$ & - & \\
\hline 3-month DASS-21 (D) & $.27 * *$ & $.53 * * *$ & $-.29 * *$ & $.26 * *$ & - \\
\hline
\end{tabular}

Note. DASS-21 (D) = Depression Anxiety and Stress Scales 21 depression scale; MPAI-4 = Mayo-Portland Adaptability Inventory $-4 ;$ SEQ = Sentinel Events Questionnaire.

$* p<.05 ; * * ; p<.01 ; * * * p<.001$

$\left(R^{2}\right.$ change $\left.=.05, p=.035\right)$ after controlling for age and discharge DASS-21 depression score. In the second analysis, total transition events significantly predicted DASS-21 depression score at 3 -months postdischarge $\left(R^{2}\right.$ change $=.04$, $p=.017)$. In the final regression analysis, total transition events and MPAI-4 change score significantly predicted DASS-21 depression score at 3-months follow-up $\left(R^{2}\right.$ change $\left.=.12, p<.001\right)$. However, only MPAI-4 change score was significant $(\beta=-.28, p=.001)$ and the effect of total transition events was reduced ( $\beta=.21$ to .14). Age and discharge DASS-21 depression score each accounted for a significant amount of variance in DASS-21 depression score at 3 -months postdischarge $(\beta=.22, p<.01 ; \beta=.48$, $p<.001)$. A Sobel test confirmed the significance of the indirect effect $(z=1.97, p<.05)$.

The pattern of total transition events and MPAI- 4 change scores was examined for the 11 individuals $(11.5 \%)$ who shifted from the normal range to the clinical range on the DASS-21 depression scale between the discharge and 3-months follow-up assessments. Consistent with the mediation findings, these individuals experienced significantly poorer progress in resuming their preinjury lifestyle (total SEQ $M=3.20, S D=1.72$ ) compared to the broader sample $(n=85, M=2.07, S D=1.35, t[94]=2.48, p<.05, d=.73)$. Furthermore, they perceived their functioning to be significantly worse on the MPAI-4 $(M=-10.73, S D=15.50)$ between discharge and 3-months follow-up, as compared to the broader sample $(M=2.36, S D=21.85, t[94]=-2.49$, $p<.05, d=.69$ ).

Follow-up analyses were conducted to assist interpretation of the relationships between the transition events, change in perceived functioning and DASS-21 depression scores. For analyses involving multiple comparisons for items on the SEQ a Bonferroni adjustment (.05/8) was applied. Discharge DASS-21 depression score was not significantly related to total transition events $(r=.10, p>.05)$ or the occurrence of individual transition events between discharge and 3-months follow-up $(p>.05)$. Only one transition event was significantly related to the DASS-21 depression score at 3-months follow-up. Specifically, individuals experiencing a lack of access to therapy reported a higher level of depressive symptoms $(M=13.20, S D=12.8)$ at 3-months postdischarge than those not experiencing this event $(M=5.25, S D=7.5$, $t[94]=-3.33, p<.006, d=.76$ ).

The MPAI-4 change score was significantly correlated with length of hospital stay $(r=-.29, p<.01)$ and PTA duration $(r=-.23, p<.05)$, but not discharge DRS $(r=-.12$, $p>.05)$ or discharge DASS-21 depression score $(r=.02$, $p>.05)$. An analysis of the association between individual transition events and MPAI-4 change scores at 3-months postdischarge revealed a trend for individuals who had returned to driving to perceived more positive changes on the

Table 4. Mediating effect of change in perceived functioning on the relationship between total transition events and depressive symptoms at 3-months postdischarge

\begin{tabular}{|c|c|c|c|c|c|}
\hline \multirow[b]{2}{*}{ Mediation analyses ${ }^{\mathrm{a}}$} & \multirow[b]{2}{*}{ Total $R^{2}$} & \multirow[b]{2}{*}{$\beta$} & \multicolumn{2}{|c|}{$95 \% \mathrm{CI}$ for B } & \multirow[b]{2}{*}{$s r^{2}$} \\
\hline & & & Lower bound & Upper bound & \\
\hline $\begin{array}{l}\text { 1. Effect of total transition events on change in perceived functioning } \\
\text { (MPAI-4 ability/adjustment change scores) }\end{array}$ & .05 & $-.22 *$ & -6.39 & -0.23 & -.22 \\
\hline $\begin{array}{l}\text { 2. Effect of total transition events on depressive symptoms } \\
\text { (3-months postdischarge) }\end{array}$ & .36 & $.21^{*}$ & 0.24 & 2.34 & .20 \\
\hline $\begin{array}{l}\text { 3. Effect of total transition events and change in perceived } \\
\text { functioning on depressive symptoms } \\
\text { (3-months postdischarge) }\end{array}$ & .43 & .14 & -0.13 & 1.92 & .14 \\
\hline $\begin{array}{l}\text { Total transition events } \\
\text { Change in perceived functioning }\end{array}$ & & $-.28 * *$ & -0.18 & -0.05 & -.27 \\
\hline
\end{tabular}

Note. MPAI-4 = Mayo-Portland Adaptability Inventory - 4.

${ }^{\mathrm{a}}$ Age and discharge depressive symptoms were controlled for in each analysis: $* p<.05, * * p<.01, * * * p<.001$. 
MPAI-4 $(M=8.55, S D=24.90)$ than those not driving $(M=-2.37, S D=19.26, t=-2.10, p<.05, d=0.49)$; however, this was not significant at the adjusted alpha level $(p>.006)$. MPAI-4 change score did not significantly differ for any other transition events $(p>.006)$.

\section{DISCUSSION}

In accordance with previous studies, approximately one quarter of the current sample reported depressive symptoms in the clinical range at both discharge and 3-months postdischarge (Kreutzer et al., 2001). Depression during early community reintegration after TBI is hence a concern for a small but not insignificant group of individuals who are at risk of poorer long-term social outcomes. Consistent with the hypothesis, the present findings indicate that transition events contributed to level of depressive symptoms at 3-months postdischarge through their influence on perceived functioning. Individuals who made greater progress in resuming their preinjury lifestyle typically perceived their abilities and adjustment to be improved at 3-months follow-up relative to discharge. Conversely, those experiencing less progress typically perceived their abilities and adjustment to be similar, or poorer, at 3-months follow-up as compared to discharge. Therefore, although discharge depressive symptoms accounted for the most variance in depressive symptoms at 3-months follow-up $\left(s r^{2}=.49\right)$, change in perceived functioning after discharge significantly predicted level of depressive symptoms during early community reintegration $\left(s r^{2}=-.27\right)$. These findings contribute to an improved conceptualization of depression following TBI in several ways.

First, by examining change scores on the MPAI-4 Ability and Adjustment indices rather than self or informant ratings at discharge (see Malec et al., 2007), this index captures the shift in individuals' subjective understanding of their postinjury functioning during early community reintegration. Second, controlling for depressive symptoms at discharge assists to distinguish early onset depressive symptoms, which are more likely to represent preexisting or neurologically based depression (Jorge et al., 2004), from reactive mood symptoms following discharge. The lack of significant associations between discharge depressive symptoms and MPAI-4 change scores and discharge depressive symptoms and transition events suggests that early onset depression is unlikely to account for the change in perceived functioning or the occurrence of transition events. The finding that only one individual transition event (i.e., lack of access to therapy) was significantly related to depressive symptoms at 3-months postdischarge indicates that the cumulative nature of transition events may be more influential than a specific event during the early community reintegration period. However, it is important to note that lack of access to therapy may present a barrier to resuming preinjury lifestyle through its association with other events (e.g., financial strain or unsuccessful attempts to return to work), rather than reflecting an independent event related to depressive symptoms. Overall, the findings support theoretical assertions that overall progress in resuming or maintaining premorbid lifestyle shapes self-understanding of postinjury limitations which, in turn, contributes to level of depressive symptoms during early community reintegration (Godfrey et al., 1993; Ownsworth \& Oei, 1998).

Given the mediating effects of MPAI-4 change scores, it is important to consider factors influencing the disparity between perceived functioning in hospital as compared to 3-months postdischarge. Consistent with theories of experience-dependent recovery (Robertson \& Murre, 1999) most of the sample perceived improved functioning at 3-months follow-up. Individuals with less severe TBI, who typically have shorter periods of hospitalization, were more likely to perceive improved functioning during the first few months postdischarge, thus suggesting that positive change scores on the MPAI-4 combined ability and adjustment index reflect actual functional gains. It is possible that those with more severe TBI (and lengthier hospitalization) have less realistic self-appraisal at discharge and begin to better understand their injury-related difficulties by 3-months postdischarge. Consequently, their change in perceived functioning was minimal, or reflected poorer functioning. This explanation is consistent with the findings of Malec et al. (2007), that individuals with moderate to severe TBI were more likely to underestimate their impairments relative to their significant other at discharge than those with mild TBI.

The present findings, combined with those of Malec et al. (2007), contribute to a preliminary model of reactive depressive symptoms during early community reintegration (see Figure 1). The model predicts that more severe TBI increases individuals' vulnerability to developing depressive symptoms after discharge through an association with perceived functioning. Individuals with severe TBI are more likely to demonstrate impaired self-awareness of functional limitations and, accordingly, report fewer depressive symptoms

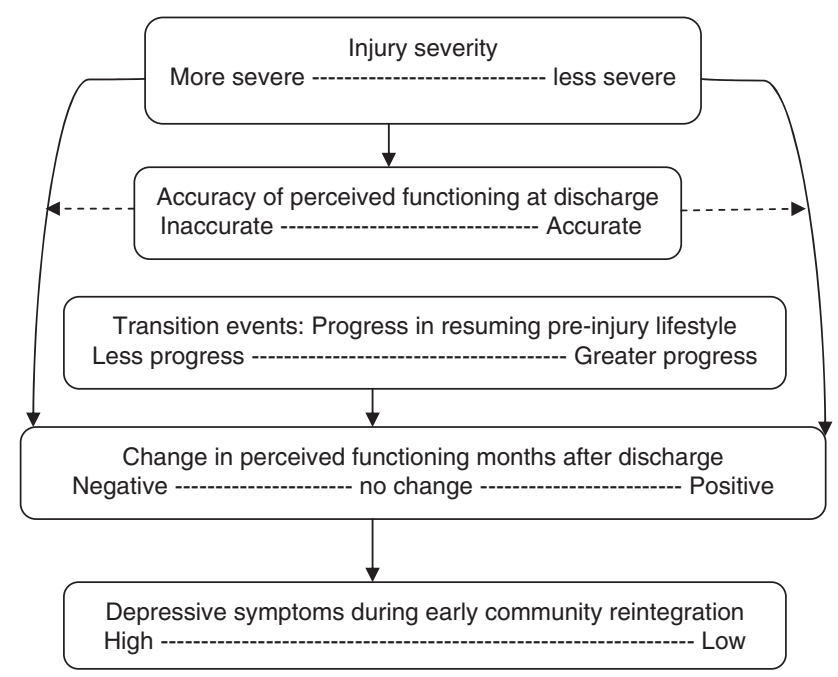

Fig. 1. A model of reactive depression during early community reintegration (3-months postdischarge) following traumatic brain injury (bold arrow lines are based on empirical findings and broken arrow lines represent a hypothesized moderating effect). 
at the time of discharge (Malec et al.). The model contends that individuals with impaired self-awareness of their functional limitations at discharge who experience greater difficulties resuming their preinjury lifestyle are likely to perceive fewer functional gains (or even poorer functioning) at 3 -months postdischarge. Consequently, these individuals may be at greater risk of developing depressive symptoms during early community reintegration (Ownsworth \& Oei, 1998). The proposed moderating effects of self-awareness at discharge on the relationship between severity of injury and change in perceived functioning after discharge requires future empirical investigation. Various other personal characteristics (e.g., appraisals, coping style, and actual functional impairments) and external factors (see Kendall \& Terry, 2009; Rutterford \& Wood, 2006) that potentially have direct or indirect effects on the relationships in the model need to be investigated in future prospective longitudinal studies to extend and validate this framework.

The main clinical implications of the present findings relate to the need for screening to identify and monitor individuals who experience adjustment difficulties related to transition and may warrant psychological intervention. Of particular concern, is the potential for a cycle of maladaptive appraisals and behaviors to develop which elevate the risk of depression postdischarge. Research by Riley, Brennan, and Powell (2004) highlighted that the experience of task difficulty or failure following brain injury can activate threat appraisals and avoidance relating to diverse everyday situations (e.g., dealing with people, leaving the house, using public transport). Although avoidance of situations may reduce anxiety in the short-term, over time such maladaptive reactions are likely to contribute to a negative self-image and the development of depression. The current findings highlight the need for individuals who are unable to resume aspects of their preinjury lifestyle to be supported to find new avenues to engage in meaningful activities and life roles (Turner et al., 2009). There is presently a lack of research evaluating interventions before or during transition that support individuals to develop more accurate understanding of their impairments and realistic plans for early community reintegration. Research by Bombardier and colleagues (2009) demonstrated that scheduled telephone-based support after discharge that primarily focuses on problem-solving and behavioral activation was effective in reducing depressive symptoms. Further evaluation of such interventions and their impact on self-appraisals, coping, emotional adjustment, and community reintegration is warranted.

It is important to acknowledge various methodological limitations in this study. First, the present findings may be less applicable to younger adults with TBI, who were less likely to consent to participate. Second, although objective data concerning global functional status were available at discharge (DRS), such data were not available at 3-months follow-up, to provide an indication of actual functional changes over this period. Third, there is some likely overlap in the constructs assessed by the DASS-21 depression scale and the MPAI-4. This is most evident on the Adjustment
Index, with item 14 describing various symptoms of depression (e.g., sad, blue, hopeless, poor appetite, poor sleep). Another likely overlap relates to the initiative (DASS-21 depression scale) and initiation (MPAI) items. However, unlike the more depression-specific items of the DASS-21, the MPAI-4 Adjustment Index more broadly reflects the common emotional, behavioral, and social consequences of TBI. Fourth, although the SEQ is a promising new approach for measuring the events that impact individuals' ability to resume their preinjury lifestyle after brain injury, further research is required to validate this tool. In the present study, the impact of transition events was examined cumulatively, to signify overall progress in resuming preinjury lifestyle. However, individuals' appraisal of the significance (or subjective weighting) of these events and their capacity to manage these is important to investigate in future research. For example, some individuals may not wish to resume particular aspects of their preinjury lifestyle (e.g., work or living independently). Such research could also examine the extent to which these events are interrelated (e.g., return to work and financial strain) and influenced by a range of preinjury, neurocognitive, and socioenvironmental factors. Finally, the applicability of the proposed model of depressive symptoms during early community reintegration needs to be investigated in larger and more culturally diverse TBI samples.

\section{CONCLUSION}

The present study investigated the relationship between transition events, perceived functioning, and the development of depressive symptoms during early community reintegration. The findings indicate that lack of progress in resuming preinjury lifestyle contributes to postdischarge depressive symptoms through an influence on perceived functioning, thus providing an improved conceptualization of reactive depression in the context of TBI. Further research is needed to extend and validate this preliminary model.

\section{REFERENCES}

Baron, R.M., \& Kenny, D.A. (1986). The moderator-mediator variable distinction in social psychological research: Conceptual, strategic and statistical considerations. Journal of Personality and Social Psychology, 51(6), 1173-1182. doi:10.1037/0022-3514. 51.6.1173

Bombardier, C.H., Bell, K.R., Temkin, N.R., Fann, J.R., Hoffman, J., \& Dikmen, S. (2009). The efficacy of a scheduled telephone intervention for ameliorating depressive symptoms during the first year after traumatic brain injury. Journal of Head Trauma Rehabilitation, 24, 230-238. doi:10.1097/HTR.0b013e3181ad65f0

Corrigan, J.D., Selassie, A.W., \& Orman, J.A. (2010). The epidemiology of traumatic brain injury. Journal of Head Trauma Rehabilitation, 25, 72-80. doi:10.1097/HTR.0b013e3181ccc8b4

Felmingham, K., Baguley, I., \& Crooks, J. (2001). A comparison of acute and postdischarge predictors of employment 2 years after traumatic brain injury. Archives of Physical and Medical Rehabilitation, 82, 435-439. doi:10.1053/apmr.2001.21985 
Godfrey, H.P.D., Partridge, F.M., Knight, R.G., \& Bishara, S. (1993). Course of insight disorder and emotional dysfunction following closed head injury: A controlled cross-sectional follow-up study. Journal of Clinical and Experimental Neuropsychology, 15, 503-515.

Gomez-Hernandez, R., Max, J.E., Kosier, T., Paradiso, S., \& Robinson, R.G. (1997). Social impairment and depression after traumatic brain injury. Archives of Physical Medicine Rehabilitation, 78, 1321-1326. doi:10.1016/S0003-9993(97)90304-X

Gracey, F., Evans, J.J., \& Malley, D. (2009). Capturing process and outcome in complex rehabilitation interventions: A "Y-shaped" model. Neuropsychological Rehabilitation, 19, 867-890. doi:10.1080/09602010903027763

Jorge, R.E., Robinson, R.G., Moser, D., Tateno, A., CrespoFacorro, B., \& Arndt, S. (2004). Major depression following traumatic brain injury, Archives of General Psychiatry, 61, 42-50. Retrieved from www.archgenpsychiatry.com

Kendall, E., \& Terry, D. (2009). Understanding the adjustment following traumatic brain injury: Is the goodness of fit coping hypothesis useful? Social Science and Medicine, 67, 1217-1224. doi:10.1016/j.socscimed.2008.05.033

Kolb, B., \& Whishaw, I.Q. (2003). Fundamentals of human neuropsychology (5th ed.). New York: Worth Publishers.

Kreutzer, J.S., Seel, R.T., \& Gourley, E. (2001). The prevalence and symptom rates of depression after traumatic brain injury: A comprehensive examination. Brain Injury, 15, 563-576.

Lovibond, S., \& Lovibond, P. (1995). Manual for the depression anxiety and stress scales (3rd ed.). Sydney: Psychology Foundation.

MacKinnon, D.P., Warsi, G., \& Dwyer, J.H. (1995). A simulation study of mediated effect measures. Multivariate Behavioural Research, 30, 41-62. doi:10.1207/s15327906mbr3001_3

Malec, J. (2005). The Mayo-Portland Adaptability Inventory. The Center for Outcome Measurement in Brain Injury. http:// www.tbims.org/combi/mpai

Malec, J.F., Testa, J.A., Rush, B.K., Brown, A.W., \& Moessner, A.M. (2007). Self-assessment of impairment, impaired selfawareness, and depression after traumatic brain injury. Journal of Head Trauma Rehabilitation, 22, 156-166. doi:10.1097/ 01.HTR.0000271116.12028.af

Nalder, E., Fleming, J., Cornwell, P., Foster, M., Worrall, L., Ownsworth, T., ... Chenoweth, L. (2010). What constitutes transition success? An investigation into factors influencing the perceptions of individuals with a TBI regarding the transition from hospital to home. Paper presented at the 8th World Congress on Brain Injury, Washington, DC.

Ownsworth, T., \& Fleming, J. (2005). The relative importance of metacognitive skills, emotional status and executive functioning in psychosocial adjustment following acquired brain injury. Journal of Head Trauma Rehabilitation, 20, 315-332.
Ownsworth, T.L., Fleming, J., Nalder, E., Cornwell, P., Haines, T., Kendall, M., \& Chenoweth, L. (2009). Occurrence of sentinel events and emotional adjustment during the hospital to home transition following acquired brain injury. Paper presented at the International Neuropsychological Society and Finnish Neuropsychological Society Joint Mid-Year Meeting, Helsinki, Finland. Abstract published in the Journal of the International Neuropsychological Society, 15(S2), 102.

Ownsworth, T.L., Little, T., Turner, B., Hawkes, A., \& Shum, D. (2008). Assessing emotional status following acquired brain injury: The clinical potential of the Depression, Anxiety and Stress Scales. Brain Injury, 22, 858-869. doi:10.1080/02699050802446697

Ownsworth, T., \& Oei, T.P.S. (1998). Depression after traumatic brain injury: Conceptualisation and treatment considerations. Brain Injury, 12, 735-751. doi:10.1080/026990598122133

Riley, G.A., Brennan, A.J., \& Powell, T. (2004). Threat appraisal and avoidance after traumatic brain injury: Why and how often are activities avoided? Brain Injury, 18, 871-888.

Robertson, I.H., \& Murre, J.M.J. (1999). Rehabilitation of brain damage: Brain plasticity and principles of guided recovery. Psychological Bulletin, 125, 544-575. doi:10.1037/0033-2909. 125.5.544

Ruff, R., Marshall, L., Crouch, M., Klauber, M., Levin, S., Barth, J., \& Marmarou, A. ... (1993). Predictors of outcome following severe head trauma: Follow-up data from the Traumatic Coma Data Bank. Brain Injury, 7, 101-111. doi:10.3109/ 02699059309008164

Rutterford, N.A., \& Wood, R.L. (2006). Evaluating a theory of stress and adjustment when predicting long-term psychosocial outcome after brain injury. Journal of the International Neuropsychological Society, 12, 359-367.

Seel, R.T., Kreutzer, J.S., Rosenthal, M., Hammond, F.M., Corrigan, J.D., \& Black, K. (2003). Depression after traumatic brain injury: A national institute on disability and rehabilitation research model systems multicenter investigation. Archives of Physical Medicine and Rehabilitation, 84, 177-184.

Tate, R.L., \& Broe, G.A. (1999). Psychosocial adjustment after traumatic brain injury: What are the important variables? Psychological Medicine, 29, 713-725. doi:10.1017/ S0033291799008466

Turner, B., Ownsworth, T.L., Cornwell, P., \& Fleming, J. (2009). Re-engagement in meaningful occupations during the transition from hospital to home for individuals with acquired brain injury and their family caregivers. American Journal of Occupational Therapy, 63, 609-620. doi:10.5014/ajot.63.5.609

Williams, W.H., \& Evans, J.J. (2003). Brain injury and emotions: An overview to a special issue on biopsychosocial approaches in neurorehabilitation. Neuropsychological Rehabilitation, 13, 1-11. doi:10.1016/S0960-9822(02)01374-X 